\title{
A triple point induced by targeted autonomization on interdependent scale-free networks
}

\author{
L D Valdez ${ }^{1,3}$, P A Macri ${ }^{1}$ and L A Braunstein ${ }^{1,2}$ \\ ${ }^{1}$ Instituto de Investigaciones Físicas de Mar del Plata (IFIMAR)-Departamento de \\ Física, Facultad de Ciencias Exactas y Naturales, Universidad Nacional de Mar del \\ Plata-CONICET, Funes 3350, (7600) Mar del Plata, Argentina \\ ${ }^{2}$ Center for Polymer Studies, Boston University, Boston, MA 02215, USA \\ E-mail: ldvaldes@mdp.edu.ar
}

Received 2 December 2013, revised 17 December 2013

Accepted for publication 18 December 2013

Published 13 January 2014

\begin{abstract}
Recent studies have shown that in interdependent networks an initial failure of a fraction $1-p$ of nodes in one network, exposes the system to a cascade of failures. Therefore it is important to develop efficient strategies to avoid their collapse. Here, we provide an exact theoretical approach to study the evolution of the cascade of failures on interdependent networks when a fraction $\alpha$ of the nodes with higher connectivity are autonomous. We found, for a pair of heterogeneous networks, two critical percolation thresholds that depend on $\alpha$, separating three regimes with very different networks' final sizes that converge into a triple point in the plane $p-\alpha$. Our findings suggest that the heterogeneity of the networks represented by high degree nodes are responsible for the rich phase diagrams found in this and other investigations.
\end{abstract}

Keywords: complex networks, percolation, branching processes PACS numbers: 64.60.aq, 64.60.ah, 89.75.Hc

(Some figures may appear in colour only in the online journal)

\section{Introduction}

Networks of networks are systems composed by several networks that in many cases depend on each other in a non-trivial way [1,2]. An example of such systems are the power grid and the communication networks [3] in which the first one provides electric power to the communication network, and the last one provides control service to the electric network. Another example is the traffic flow between cities, through the sea port and airport networks

3 Author to whom any correspondence should be addressed. 
[4] in which the flow of individuals or goods in a city decays if it does not receive traffic from one of these networks. Most of these systems are composed by individual networks connected by internal connectivity links. The role of these internal links is to generate a single component network that allows to distribute some entities between the nodes, such as the electric flow in the power grid network. Nodes of different networks are connected by interdependent links that enable the support between them. Thus in general, when a node fails in one network, the failure propagates to the other networks through the interdependent links producing sometimes a 'domino' effect with harmful consequences for the functionality of the networks.

It was shown that under a failure of a fraction of nodes in one network, the interdependence can produce a cascade of failures that spreads through all the system with catastrophic consequences in the robustness of the individual networks. Buldyrev et al [5] proposed a minimalist model, based on percolation theory, to study the dynamics of the cascade of failures. In [5] they consider two interdependent networks, denoted by $A$ and $B$ with fully interdependence i.e. each node depends on a node in the other network. By definition, a functional node is connected to the giant component (GC) of its own network and depends on a node in the other network that also belongs to its GC. Otherwise the node is dysfunctional, i.e. it is failed [5]. Thus, the GC is the only 'functional cluster' and there is only one in each network. Before receiving an initial failure or attack, every node in one network is supported by its interdependent node in the other network and thus the nodes are fully interdependent with a full correspondence between the sizes of the GC of both networks. The random failure of an initial fraction $1-p$ of nodes in one network triggers the cascade of failures and, as a consequence, the correspondence between both GC is broken. At each time step, the dysfunctional nodes transmit the failure to their interdependent neighbors, producing dysfunctional nodes in the other network. The process reaches the steady state when both networks are abruptly destroyed with a first order transition at a critical threshold $p_{c}$ or when above $p_{c}$ the correspondence between the GC is reestablished. It was shown that fully interdependent networks are very fragile under random failures, i.e., they have a higher critical threshold $p_{c}$ than isolated networks, regardless of the degree distribution [5]. This was an exciting result because it is well known that isolated heterogeneous scale-free $(\mathrm{SF})$ networks are very robust against random failures $\left(p_{c} \rightarrow 0\right)$. However, to consider full interdependence is not very realistic because nodes in each network can work autonomously. For example, some nodes can have a 'power supply' or a backup that allow them to remain functional even when they lack of support from the other network, increasing their chance to remain functional. As a consequence, partial interdependence where a random fraction $q<1$ of nodes are interdependent and the rest are autonomous, increases the robustness of the individuals networks compared to the case of fully interdependence [6-10]. It was found that depending on the value of $q$ and on the fraction $1-p$ of random failures in network $A$, the transition changes from a discontinuous to a continuous one. In these partial interdependent networks the correspondence in the steady state between networks $A$ and $B$ is broken because the autonomous nodes in network $B$ do not receive the initial failure of network $A$ and can only become dysfunctional by the failure of non-autonomous nodes that disconnect them from its GC. Then, in the steady state, the size of the GC of network $B$ is bigger than the one in network $A$. In [7] it was found that for heterogeneous SF under random autonomization the sizes of the functional clusters undergo an abrupt decreasing for a certain value of $p=p_{c}^{+}$without a full collapse due to the fact that the high degree nodes are sustained by autonomous nodes ${ }^{4}$. However bellow $p_{c}^{+}$the size of the GC of network $A$ decreases

4 Note that since in a SF network there is only a small amount of high degree nodes, they have a low probability to become dysfunctional in the initial failure. 
continuously to zero as in a second order percolating transition at a value $p=p_{c}^{-}$, while the size of network $B$ goes to a finite value. The goal is to find a way to autonomize efficiently the networks in order to increase their robustness compared to the case of random autonomization $[11,12]$. Schneider et al [11] proposed a model where the robustness of the system is enhanced by targeted autonomization of a fraction $\alpha \equiv 1-q$ of the higher degree nodes. Using a theoretical mean field approximation that assumes that the cascade of failures affects both autonomous and non-autonomous nodes, they showed that even for homogeneous networks there is a critical point in the plane $p-q$, at $\left(p_{c}, q_{c}\right)$ where the transition changes from first order for $q>q_{c}$ to a continuous one for $q<q_{c}$. This theoretical result was qualitatively supported by simulation, but the exact theoretical solution was not derived so far. An exact theoretical formulation allows to find some other effects that are hidden in the simulations due to finite size effects. Very recently, Valdez et al [13], introduce an exact general framework that they apply to explain the effect of partially correlated interdependent networks in the robustness of heterogeneous SF interdependent networks under cascade of failures. The exact result allowed to find very interesting features such as a triple point in the phase diagram that depend on the level of correlation. Here, we apply the formalism presented in [13] to targeted autonomization and derive the exact theoretical solutions for this process.

\section{Theoretical results}

We study the temporal evolution of the sizes of the GC of two interdependent networks under targeted autonomization when a fraction $\alpha$ of the higher degree nodes of both networks are autonomous. Each network, that we denote by $A$ and $B$, has connectivity links distributed according to $P\left[k_{A}\right]$ and $P\left[k_{B}\right]$, where $k_{A}$ and $k_{B}$ are the connectivity links of nodes in $A$ and $B$ respectively. Let us assume that a fraction of interdependent nodes $q_{A}\left[k_{A}\right]\left(q_{B}\left[k_{B}\right]\right)$ in network $A(B)$ depends on the connectivity links of network $A(B)$. In the initial stage a fraction $1-p$ of nodes fails at random in network $A$. At each stage $n$ of the cascade failure that goes from $A$ to $B$, a node in network $A$ with degree $k_{A}$ is functional if it is autonomous and belongs to its GC with probability $\left(1-q_{A}\left[k_{A}\right]\right)\left(1-\left(1-p f_{A n}\right)^{k_{A}}\right)$ or if it is not autonomous but was connected to the GC of $B$ in a previous stage with probability $q_{A}\left[k_{A}\right]\left(1-\left(1-f_{B n-1}\right)^{k_{B}}\right)$. Since the initial failure (at $n=0$ ) of $1-p$ nodes happens only in network $A$, then only $f_{A n}$ is multiplied by $p$. Here $f_{A n}\left(f_{B n}\right)$ is the probability that a random selected edge that leads to a non-failed node at $n=0$, this node belongs to the GC of network $A(B)$ at stage $n[14,15]$ and fulfils the self-consistent equation

$$
\begin{aligned}
f_{A n}= & \sum_{k_{A}=k_{\min }}^{k_{\max }} \frac{k_{A} P\left[k_{A}\right]}{\left\langle k_{A}\right\rangle}\left(1-q_{A}\left[k_{A}\right]\right)\left(1-\left(1-p f_{A n}\right)^{k_{A}-1}\right) \\
& +\sum_{k_{A}=k_{\min }}^{k_{\max }} \frac{k_{A} P\left[k_{A}\right]}{\left\langle k_{A}\right\rangle} q_{A}\left[k_{A}\right]\left(1-\left(1-p f_{A n}\right)^{k_{A}-1}\right) \\
& \times \sum_{k_{B}=k_{\min }}^{k_{\max }} P\left[k_{B}\right]\left(1-\left(1-f_{B n-1}\right)^{k_{B}}\right)
\end{aligned}
$$

where $k_{\min }$ and $k_{\max }$ are the minimum and maximum connectivity links respectively.

The first term in equation (1) takes into account the autonomous functional nodes in $A$ with degree $k_{A}$ and the second term corresponds to functional nodes in $A$ with degree $k_{A}$ that depend on functional nodes of $B$ with degree $k_{B}$ at step $n-1$. Thus, the fraction of nodes $\Psi_{n}$ 
of the GC of network $A$ at step $n$ is given by,

$$
\begin{gathered}
\Psi_{n}=p\left(\sum_{k_{A}=k_{\min }}^{k_{\max }} P\left[k_{A}\right]\left(1-q_{A}\left[k_{A}\right]\right)\left(1-\left(1-p f_{A n}\right)^{k_{A}}\right)+\sum_{k_{A}=k_{\min }}^{k_{\max }} q_{A}\left[k_{A}\right] P\left[k_{A}\right]\left(1-\left(1-p f_{A n}\right)^{k_{A}}\right)\right. \\
\left.\quad \times \sum_{k_{B}=k_{\min }}^{k_{\max }} P\left[k_{B}\right]\left(1-\left(1-f_{B n-1}\right)^{k_{B}}\right)\right)
\end{gathered}
$$

For network $B, f_{B n}$ also fulfils a self-consistent equation

$$
\begin{aligned}
f_{B n}= & \sum_{k_{B}=k_{\min }}^{k_{\max }} \frac{k_{B} P\left[k_{B}\right]}{\left\langle k_{B}\right\rangle}\left(1-q_{B}\left[k_{B}\right]\right)\left(1-\left(1-f_{B n}\right)^{k_{B}-1}\right) \\
& +p \sum_{k_{B}=k_{\min }}^{k_{\max }} \frac{k_{B} P\left[k_{B}\right]}{\left\langle k_{B}\right\rangle} q_{B}\left[k_{B}\right]\left(1-\left(1-f_{B n}\right)^{k_{B}-1}\right) \\
& \times \sum_{k_{A}=k_{\min }}^{k_{\max }} P\left[k_{A}\right]\left(1-\left(1-p f_{A n}\right)^{k_{A}}\right) .
\end{aligned}
$$

Thus the fraction of nodes $\phi_{n}$ of the GC of network $B$ is given by

$$
\begin{aligned}
\phi_{n}= & \sum_{k_{A}=k_{\min }}^{k_{\max }} P\left[k_{B}\right]\left(1-q_{B}\left[k_{B}\right]\right)\left(1-\left(1-f_{B n}\right)^{k_{B}}\right) \\
& +p \sum_{k_{A}=k_{\min }}^{k_{\max }} P\left[k_{A}\right]\left(1-\left(1-p f_{A n}\right)^{k_{A}}\right) \\
& \times \sum_{k_{B}=k_{\min }}^{k_{\max }} q\left[k_{B}\right] P\left[k_{B}\right]\left(1-\left(1-f_{B n}\right)^{k_{B}}\right) .
\end{aligned}
$$

In the steady state, i.e. for $n \rightarrow \infty, \Psi_{n} \approx \Psi_{n-1}$ and $\phi_{n} \approx \phi_{n-1}$, thus $\Psi_{n}$ and $\phi_{n}$ converges to $\Psi_{\infty}$ and $\phi_{\infty}$, respectively [13, 16, 17].

In this model, $q_{A}\left[k_{A}\right]$ and $q_{B}\left[k_{B}\right]$ are given by

$$
q_{i}\left[k_{i}\right]= \begin{cases}1 & k_{i}<k_{S} \\ (1-w) & k_{i}=k_{S} \\ 0 & k_{S}<k_{i}\end{cases}
$$

where $i=A, B, k_{S}$ is the degree at and above which a fraction $\alpha$ of nodes are autonomous, and $k_{S}$ fulfils $\sum_{k_{i}=k \min }^{k_{s}-1} P\left[k_{i}\right] \leqslant 1-\alpha<\sum_{k_{i}=k \min }^{k_{s}} P\left[k_{i}\right]$. Thus if we denote by $w$ the fraction of autonomous nodes with degree $k_{s}, w P\left[k_{s}\right]+\sum_{k_{i}=k_{s}+1}^{k_{\max }} P\left[k_{i}\right]=\alpha$. Note that for $\alpha>0$ equations (1)-(4) are not symmetric which leads to the non-correspondence between the final sizes of the GC of networks $A$ and $B$, as mentioned above. The symmetry is restored only for $q_{A}=q_{B}=1(\alpha=0)$ [5] or when the initial failure happens in both networks [7].

We apply our equations to SF networks of sizes $N=10^{6}$ with degree distribution $P[k] \sim k^{-\lambda}$ with $k_{\min } \leqslant k \leqslant k_{\max }$ and $\lambda=2.5$. Here, we use $k_{\min }=2$ to ensure that at the beginning all the nodes belong to the GC [18]. Since the theoretical solutions of equations (1)-(4) near the criticality are sensitive to the precision employed in the calculations, we use a multiple precision arithmetic library [19]. We chose a finite $k_{\max }$ in order to emulate the finite power law region observed in many real networks [20] such as the movie actor network [21], the scientific collaboration network [15, 22] and the protein network [23]. In figure 1 we show 

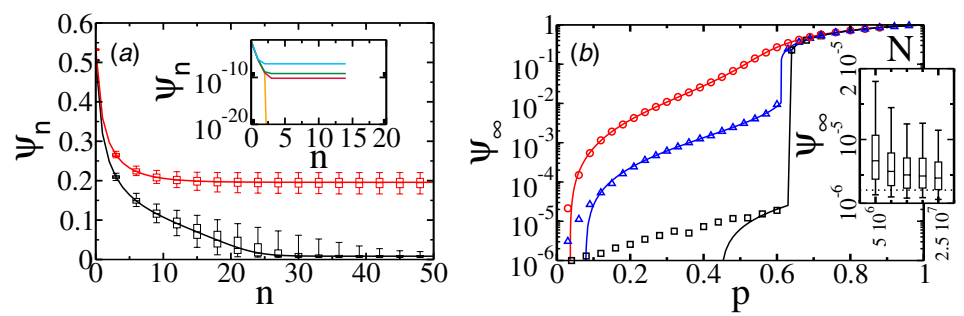

Figure 1. Cascade failure on network $A$ with targeted autonomization on SF networks of size $N=10^{6}$ with $\lambda=2.5$ and $2 \leqslant k \leqslant k_{\max }=1000$. (a) $\Psi_{n}$ as a function of $n$ obtained by 100 network realizations (box plots) and from equations (1)-(4) (solid lines) for $\alpha=0.01 \%, p=0.60$ (black) and $p=0.62$ (red). The ends of the whiskers represent the 5th-percentile and 95th-percentile. In the inset we plot the theoretical solution for (from top to bottom): $p=0.07790, p=0.077822, p=0.0778214$ and $p=0.0778213\left(p_{c} \approx 0.077821334\right)$. (b) $\Psi_{\infty}$ as a function of $p$ for different values of $\alpha, \alpha=0.1 \%$ (red, $\bigcirc$ ), $\alpha=0.01 \%$ (blue, $\Delta$ ) and $\alpha=0.001 \%$ (black, $\square$ ). The simulations are represented by symbols and the theoretical solutions obtained from equations (1)-(4) for $n \rightarrow \infty$ by solid lines. The simulations were performed over 100 network realizations. In the inset we show the finite size effects of the simulations as $N$ increases from $N=5 \times 10^{6}$ to $N=2.5 \times 10^{7}$ for $p=0.47$ and $\alpha=0.001 \%$. Each box plot is obtained over 1000 network realizations and shows the 5th, 25th, 50th, 75th and 95th percentile values. For bigger network sizes, the median of $\Psi_{\infty}$ approaches to the theoretical value (dotted line).

the temporal evolution and the steady state of the fraction of nodes in the GC of network $A$, for SF networks with $\lambda=2.5$. From figure 1 we can see the excellent agreement between the theory and the simulations, except for very low values of $\Psi_{\infty}$ that is due to finite size effects. In the inset of figure1 $(b)$ we show from $N=5 \times 10^{6}$ to $N=2.5 \times 10^{7}$ that the value of $\Psi_{\infty}$ obtained from the simulations approaches to the theoretical solution for $p=0.47$ and $\alpha=0.001 \%$ as the system size increases.

As was observed in [11], for homogeneous networks, the robustness of the networks increases with $\alpha$ due to the fact that the higher degree nodes of both networks, that are the ones that sustain the functionality of the networks, are autonomous. Our theoretical equations allow one to find a surprising behavior of the transitions with two critical thresholds at $p=p_{c}^{+}$and $p=p_{c}^{-}$that depend on $\alpha$ (with $p_{c}^{-}<p_{c}^{+}$). At $p_{c}^{+}$the sizes of the functional networks $A$ and $B$ have an abrupt jump. Below this critical threshold the GC of network $A$ is destroyed at $p_{c}^{-}$. To compute the value of the critical point $p_{c}^{+}$, we solve numerically the system of equations (1) and (3) with the condition $\operatorname{det}(J-I)=0,{ }^{6}$ where $J$ is the Jacobian of equations (1) and (3) and $I$ is the identity matrix. This method also can be applied to find $p_{c}^{-}$, however here we use a more explicit and physical derivation to compute $i t^{7}$. Assuming that the transition in network $A$ is continuous, then the probability $f_{A} \rightarrow 0$ continuously when $p \rightarrow p_{c}^{-}$. As a consequence at this threshold $f_{B}$, that is not zero due to the broken symmetry imposed by the initial failure

5 In [11] it only one threshold was found, since the process was studied only on homogeneous networks.

6 Geometrically, this equation is the condition of the tangency between the identity plane and the surface composed by the right hand side of equations (1) and (3) in the steady state.

7 Another method to obtain graphically the value of the critical point is by measuring the position of the peak of the NOI curve as a function of $p$, where the NOI is the number of iterations needed to reach the steady state of the evolution equations. At $p_{c}^{+}$there is a sharp peak, which corresponds to the condition $\operatorname{det}(J-I)=0$. However, around $p_{c}^{-}$the NOI has not a visible peak within the precision we used. 


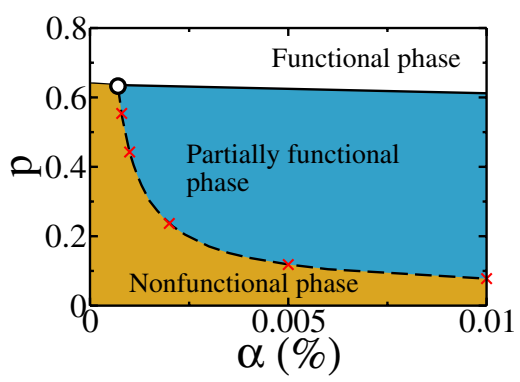

Figure 2. Phase diagram in the plane $\alpha-p$ : (i) the yellow area corresponds to the nonfunctional phase, i.e., $\Psi_{\infty}=0$, (ii) the blue area corresponds to a partial functional phase in which the size of the GC of both networks is $\lesssim 10^{-3}$ and (iii) the white area corresponds to a functional phase where $\Psi_{\infty} \gtrsim 10^{-2}$. The white circle corresponds to a triple point. The solid lines represent the abrupt change on both network's sizes and the dotted line, which is defined for $\alpha>\alpha_{c}$, represents a continuous transition of $\Psi_{\infty}$ at $p_{c}^{-}$, obtained from equation (7). The cross symbols correspond to some points obtained from equations (1)-(4) around which the solution $\Psi_{n}$ vanishes for $n \rightarrow \infty$.

in $A$ and by the partially interdependence $(0<\alpha<1)$, reduces to

$$
f_{B}=\sum_{k_{B}=k_{\min }}^{k_{\max }} \frac{k_{B} P\left[k_{B}\right]}{\left\langle k_{B}\right\rangle}\left(1-q_{B}\left[k_{B}\right]\right)\left(1-\left(1-f_{B}\right)^{k_{B}-1}\right) .
$$

Solving this self-consistent equation we found the non-trivial solution of equation (5), from where we obtain $f_{B}$ at the threshold $p_{c}^{-}$. Since $p_{c}^{-}$is a critical point for network $A$, the rhs of equation (1) for $n \rightarrow \infty$ is tangent to the identity function evaluated at $f_{A}=0$, thus

$$
\begin{aligned}
1= & p \sum_{k_{A}=k_{\min }}^{k_{\max }} \frac{k_{A}\left(k_{A}-1\right) P\left[k_{A}\right]}{\left\langle k_{A}\right\rangle}\left(1-q_{A}\left[k_{A}\right]\right) \\
& +p \sum_{k_{A}=k_{\min }}^{k_{\max }} q_{A}\left[k_{A}\right] \frac{k_{A}\left(k_{A}-1\right) P\left[k_{A}\right]}{\left\langle k_{A}\right\rangle} \sum_{k_{B}=k_{\min }}^{k_{\max }} P\left[k_{B}\right]\left(1-\left(1-f_{B}\right)^{k_{B}}\right) .
\end{aligned}
$$

Then $p=p_{c}^{-}$is explicitly given by

$p_{c}^{-}=\left[\frac{\left\langle k_{A}^{2}\right\rangle-\left\langle k_{A}\right\rangle}{\left\langle k_{A}\right\rangle}-G_{0 B}\left[1-f_{B}\right]\left(\sum_{k_{A}=1}^{k_{s}-1} \frac{k_{A}\left(k_{A}-1\right) P\left[k_{A}\right]}{\left\langle k_{A}\right\rangle}+\frac{(1-w) k_{s}\left(k_{s}-1\right) P\left[k_{s}\right]}{\left\langle k_{A}\right\rangle}\right)\right]^{-1}$,

where $\left(\left\langle k_{A}^{2}\right\rangle-\left\langle k_{A}\right\rangle\right) /\left\langle k_{A}\right\rangle$ is the branching factor of random percolation in network $A$ and $G_{0 B}[x] \equiv \sum_{k_{B}} P\left[k_{B}\right] x^{k_{B}}$ is the generating function of network $B$. Thus $p_{c}^{-}$is a correction to the threshold of percolation in individual networks where $p_{c}=\left\langle k_{A}\right\rangle /\left(\left\langle k_{A}^{2}\right\rangle-\left\langle k_{A}\right\rangle\right)$, because the branching factor in this process is reduced by the second term, as a result of the targeted autonomization. Note that if $k_{\max } \rightarrow \infty$ the branching factor diverges, and $p_{c}^{-} \rightarrow 0$ for all $\alpha>0 .{ }^{8}$ The solution of equation (7), has a physical meaning only if $p_{c}^{-}<p_{c}^{+}$, otherwise there is only one threshold at $p=p_{c}^{+}$where both networks fully collapses. The phase diagram in the plane $p-\alpha$, displayed in figure 2 , shows a triple point in which the line of the first order

8 It is straightforward to obtain the same result for random autonomization [7] under random failure in both networks. 
transition forks at $\alpha_{c}=0.000702$ (1)\% into two branches where the upper one corresponds to an abrupt collapses at $p=p_{c}^{+}$and the lower one corresponds to $p_{c}^{-}$where the size of network $A$ continuously vanishes.

If the assumption on the continuity of the transition used to derive $p_{c}^{-}$holds, the evolution equation around $p_{c}^{-}$will also show a continuous critical behavior at the value of $p_{c}^{-}$obtained from equation (7). We solve numerically the equations (1)-(4) for $p_{c}^{-}+\delta p$, for different values of $\alpha$. In the inset of figure 1(a) we show the temporal evolution for $\alpha=0.01 \%$ (with $p_{c}^{-}=0.077821334$ ). We can see that above but very close to our theoretical $p_{c}^{-}, \Psi_{\infty}$ goes to a finite value, while slightly below network $A$ collapses $\left(\Psi_{\infty}=0\right)$. In figure 2 we show (with cross symbols) some values of $p_{c}^{-}$of the continuous branch of the phase diagram obtained from the evolution equations ${ }^{9}$, that are in total agreement with equation (7). This result confirms our argument which leads to equation (7), used to obtain the lower branch of the phase diagram allowing us to find $p_{c}^{-}$. We found the same qualitative behavior for different values of $\lambda$, however as the heterogeneity decreases, the network is less robust and it is expected that at some point the triple point will be lost. At this point the phase diagram will have only one transition line, such as in homogeneous networks [11]. These findings may indicate that when high degree nodes in SF networks are protected via targeted autonomization, random autonomization [7] or correlation [13], they induce multiple and different kinds of order transitions.

In summary, we have presented the exact formulation of the cascade of failures for targeted immunization with any degree distribution of connectivity links. We show theoretically that increasing autonomization $\alpha$ enhances the robustness of SF networks and generates in the phase diagram $p-\alpha$ different regimes with different characteristic sizes of the GC. These regimes converge into a triple point which is reminiscent of the triple points of liquids. Physically it means that high degree nodes, that are responsible for maintaining the integrity of the networks, play a fundamental role in the rich phase diagrams of these processes.

\section{Acknowledgments}

LDV, PAM and LAB thank UNMdP and FONCyT (Pict 0293/2008) for financial support.

\section{References}

[1] Rinaldi S M, Peerenboom J P and Kelly T K 2001 Identifying, understanding, and analyzing critical infrastructure interdependencies IEEE Control Syst. 21 11-25

[2] Kivelä M, Arenas A, Barthelemy M, Gleeson J P, Moreno Y and Porter M A 2013 Multilayer networks arXiv:1309.7233

[3] Rosato V, Issacharoff L, Tiriticco F, Meloni S, Porcellinis S and Setola R 2008 Modelling interdependent infrastructures using interacting dynamical models Int. J. Crit. Infrastruct. 4 63-79

[4] Parshani R, Rozenblat C, Ietri D, Ducruet C and Havlin S 2010 Inter-similarity between coupled networks Europhys. Lett. 9268002

[5] Buldyrev S V, Parshani R, Paul G, Stanley H E and Havlin S 2010 Catastrophic cascade of failures in interdependent networks Nature $\mathbf{4 6 4} 1025-8$

[6] Parshani R, Buldyrev S V and Havlin S 2010 Interdependent networks: reducing the coupling strength leads to a change from a first to second order percolation transition Phys. Rev. Lett. 105048701

[7] Zhou D, Gao J, Stanley H E and Havlin S 2013 Percolation of partially interdependent scale-free networks Phys. Rev. E 87052812

9 In order to determine $p_{c}^{-}$, we use $\delta p=10^{-12}$. 
[8] Gao J, Buldyrev S V, Havlin S and Stanley H E 2011 Robustness of a network of networks Phys. Rev. Lett. 107195701

[9] Gao J, Buldyrev S V, Stanley HE and Havlin S 2011 Networks formed from interdependent networks Nature Phys. 8 40-48

[10] Gao J, Buldyrev S V, Stanley H E, Xu X and Havlin S 2013 Percolation of a general network of networks arXiv:1306.3416

[11] Schneider C M, Yazdani N, Araújo N A M, Havlin S and Herrmann H J 2013 Towards designing robust coupled networks Sci. Rep. 31969

[12] Yagan O, Qian D, Zhang J and Cochran D 2012 Optimal allocation of interconnecting links in cyber-physical systems: interdependence, cascading failures, and robustness IEEE Trans. Parallel Distrib. Syst. 23 1708-20

[13] Valdez L D, Macri P A, Stanley HE and Braunstein L A 2013 Triple point in correlated interdependent networks Phys. Rev. E 88 050803(R)

[14] Braunstein L A, Wu Z, Chen Y, Buldyrev S V, Kalisky T, Sreenivasan S, Cohen R, López E, Havlin S and Stanley H E 2007 Optimal path and minimal spanning trees in random weighted networks Int. J. Bifurcation Chaos 17 2215-55

[15] Newman M E J, Strogatz S and Watts D 2001 Random graphs with arbitrary degree distributions and their applications Phys. Rev. E 64026118

[16] Son S-W, Bizhani G, Christensen C, Grassberger P and Paczuski M 2012 Percolation theory on interdependent networks based on epidemic spreading Europhys. Lett. 9716006

[17] Baxter G J, Dorogovtsev S N, Goltsev A V and Mendes J F F 2012 Avalanche collapse of interdependent networks Phys. Rev. Lett. 109248701

[18] Bornholdt S, Schuster H G and Wiley J 2003 Handbook of Graphs and Networks vol 2 (New York: Wiley)

[19] Nikolaevskaya E, Khimich A and Chistyakova T 2012 Programming with Multiple Precision vol 397 (Berlin: Springer)

[20] Nunes Amaral L A, Scala A, Barthélémy M and Stanley H E 2000 Classes of small-world networks Proc. Natl Acad. Sci. 9711149

[21] Barabási A-L and Albert R 1999 Emergence of scaling in random networks Science 286 509-12

[22] Barabási A-L, Jeong H, Néda Z, Ravasz E, Schubert A and Vicsek T 2002 Evolution of the social network of scientific collaborations Physica A 311 590-614

[23] Jeong H, Mason S P, Barabási A-L and Oltvai Z N 2001 Lethality and centrality in protein networks Nature 411 41-42 